\title{
Control of the MARES Autonomous Underwater Vehicle
}

\author{
Bruno Ferreira, Miguel Pinto, Aníbal Matos, Nuno Cruz \\ FEUP - DEEC \\ Rua Dr. Roberto Frias, s/n 4200-465 Porto PORTUGAL \\ ee04018@fe.up.pt, ee04134@fe.up.pt, anibal@fe.up.pt, nacruz@fe.up.pt
}

\begin{abstract}
This paper focuses the control problem of a nonholonomic autonomous underwater vehicle, moving in the tridimensional space. The dynamic of a body in submarine environments is strongly nonlinear. This implies that classical linear controllers are often inadequate whereby Lyapunov theory is here considered. Methods based in this theory are promising tools to design controllers and are applied to the case of MARES, a small-sized autonomous underwater vehicle. Several controllers based only on Lyapunov theory are determined while others combine linear and nonlinear control theory in order to perform various maneuvers. Aiming to verify the correct performance of controllers, simulations and experiments are carried out.
\end{abstract}

\section{INTRODUCTION}

\section{A. MARES AUV}

MARES, or Modular Autonomous Robot for Environment Sampling [1-2], is a $1.5 \mathrm{~m}$ long AUV (Autonomous Underwater Vehicle), designed and built by the Ocean Systems Group at the Faculty of Engineering of University of Oporto. MARES has a slender body form and is endowed with four thrusters that confer it four controllable degrees of freedom (DOF). Each thruster may reach forces around $21.5 \mathrm{~N}$. It can dive up to $100 \mathrm{~m}$ deep, and unlike similar-sized systems, has vertical thrusters to allow for purely vertical motion in the water column. Forward velocity may be independently defined, from 0 to about $1.5 \mathrm{~m} / \mathrm{s}$ by regulation of horizontal thruster forces.

Though MARES can have multiple configurations, we will only assume the presented in fig. 1 .

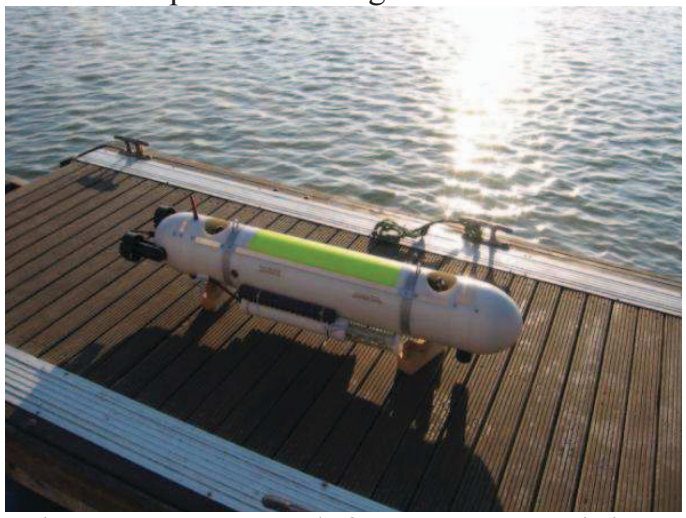

Fig. 1: MARES AUV ready for an autonomous mission.

\section{B. Dynamic}

Every body inserted in a fluid is experiences several forces during its motion [3-5]. For submerged bodies, these forces are essentially:

- Added mass forces, originated by the acceleration of involving particles of fluid during the acceleration of the body;

- Drag forces, due to friction and pressure on the hull and vortices created by non null velocity;

- Froude-Krylov force due to the acceleration of the fluid;

- Restoring forces due to the weight and to the buoyancy;

- Propulsion forces exercised by actuators (thrusters).

The resulting dynamic is highly nonlinear and depends on relative velocity and absolute position. In addition, the motion in six degrees of freedom turns the dynamic and the control problem more complex.

\section{Control}

The existence of referred forces confers to submerged body a highly nonlinear behavior during its motion. This fact implies that classical linear control may not be adequate for the referred dynamic. Though it is used by some authors for underwater vehicles, as [3] and [6], the system response tends to degrade in a large range of operation or even to turn instable.

In addition, the complexity of the control problem increases when the vehicle moves in three dimensions. An interesting approach is proposed by [7] for underactuated vehicles path following.

In this paper, we intend to design controllers for the nonholonomic vehicle MARES. These should allow performing several maneuvers in the tridimensional space.

\section{BACKGROUND}

\section{A. Kinematic}

In some robotic application it is useful to express different vectors in different coordinate systems [8]. For convenience, we define two referential. One of them must be fixed to earth and considered inertial. The second one is fixed to the vehicle. This allows defining vectors of the position and of the velocity related to fluid, respectively:

$$
\eta=\left[\begin{array}{llllll}
x & y & z & \phi & \theta & \psi
\end{array}\right]^{T},
$$




$$
v_{r}=\left[\begin{array}{llllll}
u & v & w & p & q & r
\end{array}\right]^{T} .
$$

The first three components of each vector are linear components and second ones are angular. $\eta$ is given in the earth-fixed referential while $v_{r}$ is given in the body-fixed one. The fig. 2 illustrates presented concepts.

Both referential may be related by the rotation matrix $J(\phi, \theta, \psi) \in \mathbb{R}^{3 \times 3}$. A linear vector $c_{B}$ expressed in the bodyfixed referential may be expressed in the earth-fixed one, through the following relation:

$$
c_{E}=J \cdot c_{B}
$$

The rotation matrix is orthonormal, which implies that $J^{-1}=J^{T}$. It is important to refer that this matrix may be obtained by decomposition of elementary rotations such that

$$
J(\phi, \theta, \psi)=J(\phi, 0,0) \cdot J(0, \theta, 0) \cdot J(0,0, \psi)
$$

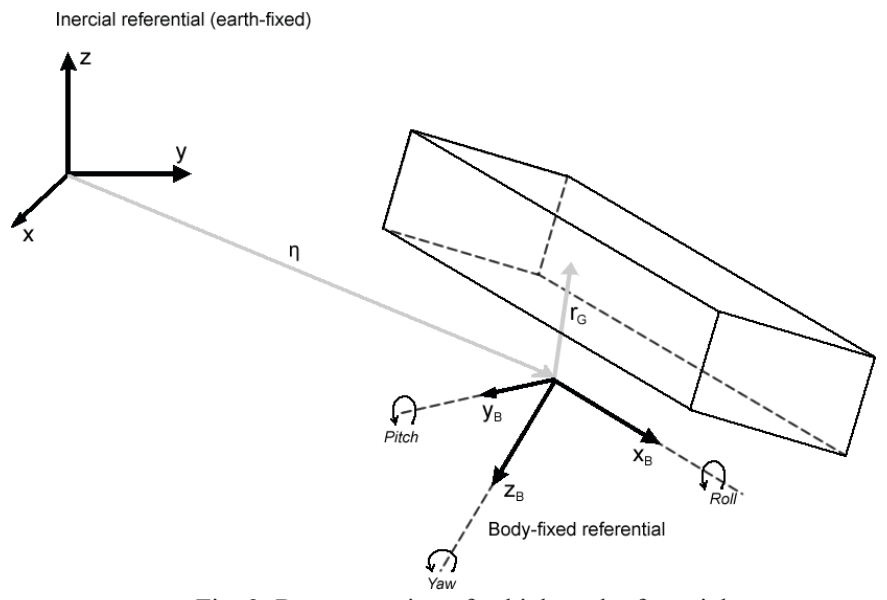

Fig. 2: Representation of vehicle and referential

\section{B. Model of MARES}

Through motion modeling, we get a general equation of MARES dynamic, as follow:

$$
\begin{aligned}
\left(M_{R B}+M_{A}\right) \dot{v}_{r} & =-\left(C_{R B}\left(v_{r}\right)+C_{A}\left(v_{r}\right)\right. \\
& \left.+D_{V}\left(v_{r}\right)\right) \cdot v_{r}-g(\eta)+P f_{p},
\end{aligned}
$$

where $M_{R B}, M_{A}, C_{R B}, C_{A}, D_{V} \in \mathbb{R}^{6 \times 6}$ are, respectively, the rigid body inertia, the added mass inertia, the Coriolis and centripetal terms of rigid body, the Coriolis and centripetal terms of added mass and the viscous damping matrices. $v_{r} \in \mathbb{R}^{6}$ is the velocity vector, $\dot{v}_{r}$ its time derivative, $\eta$ the position vector, $g(\eta) \in \mathbb{R}^{6}$ the restoring forces and moments vector, $P \in \mathbb{R}^{6 \times 4}$ the actuation matrix and $f_{p} \in \mathbb{R}^{4}$ the generated thruster force vector.

We consider that the fluid acceleration is small in locals where missions are performed, whereby Froude-Krylov forces are negligible. Remaining forces depend on geometric, mass and buoyancy characteristics, as it can be verified in [3], [6] and [9].

\section{Lyapunov direct method}

Lyapunov theory is often used in nonlinear systems as in [7]. It allows concluding about the stability of a system and designing control laws. Authors recommend the reading of [10] and [11] for a more detailed presentation of the Lyapunov theory.
Lyapunov direct method is based on the analysis of energy behavior in a system. The major principle is reasoned by the following fact: if the total energy of a system is constantly dissipated along its operation, or motion, it will stabilize in an equilibrium or point, or state. To illustrating this idea, we consider an oscillating pendulum with non null potential energy. Assuming that there exists friction due to oscillation, the amplitude of its motion will reduce gradually until stop in the point where the resultant force is zero (equilibrium point where the potential energy is lower).

Before presenting the more useful results from this theory, we must define concepts of positive and negative definite:

- A scalar function $f(x)$ is said to be globally positive definite if $f(x)>0, \forall x \neq 0$ and globally positive semi-definite if $f(x) \geq 0, \forall x \neq 0$;

- A scalar function $f(x)$ is said to be globally negative definite if $f(x)<0, \forall x \neq 0$ and globally negative semi-definite if $f(x) \leq 0, \forall x \neq 0$.

A function $V(x)$ is said to be a Lyapunov function of a system if it is positive definite, with continuous partial first derivatives and, in addition, if its time derivative is negative semi-definite $(\dot{V}(x) \leq 0, \forall x \neq 0)$ for any trajectory of the state $x$.

Lyapunov theory states through the global stability theorem that if "there exists a scalar function $V$ of the state $x$ with continuous first derivatives such that $V(x)$ is positive definite, $\dot{V}(x)$ is negative definite and $V(x) \rightarrow \infty$ as $x \rightarrow \infty$, then the equilibrium point at the origin is globally asymptotically stable" [10].

\section{CONTROL}

In order to perform some maneuvers, it is necessary an adequate control of MARES AUV. Using Lyapunov theory, we will determine several controllers. In this section, we start by presenting the development of vertical and horizontal velocity, prosecuting with position controllers. These last will allow that the vehicle remain in a determined vertical position, follow an imaginary line or circle and stay immobile in the water column.

The model given in the section II allows obtaining an approach to the real behavior of MARES, however it is affected by modeling uncertainties and neglected terms. For the development of controllers, these deviations are considered disturbances during the operation of the vehicle.

The control of MARES is realized by actuations of thrusters, whereby, from expression (4), we may conclude that $f_{p}$ is the control variable. We assume that we can instantly vary the forces of propellers though it is not true in reality. This assumption simplifies the analysis and the determination of controllers and can be justified by the fact that time constants associated to the actuation are much smaller than the ones associated to the vehicle motion. Some controllers are developed combining nonlinear and linear [12] controllers.

\section{A. Vertical velocity controller}

In this subsection we will only consider the motion in the vertical plane or, in other words, in the $z_{B}$-axis direction and in 
the pitch angle (see fig. 3). To simplify the determination of the vertical velocity controller, we start by reducing the order of the model. This implies that we must eliminate lines and columns of matrices of equation (4) whose influences in the $Z_{B}$ and pitch motion are negligible. It results on the elimination of second, fourth and sixth lines and columns of matrices in (4). In the vectors case, only the same lines are eliminated. Note that the forward velocity $u$ (after $x_{B}$-axis) is considered in this case because the model has non negligible cross terms that influence the vertical motion, as it can be seen in [9]. Neglecting this velocity component would not be critical but it would generate a less robust response of the system to disturbances due to forward velocity.

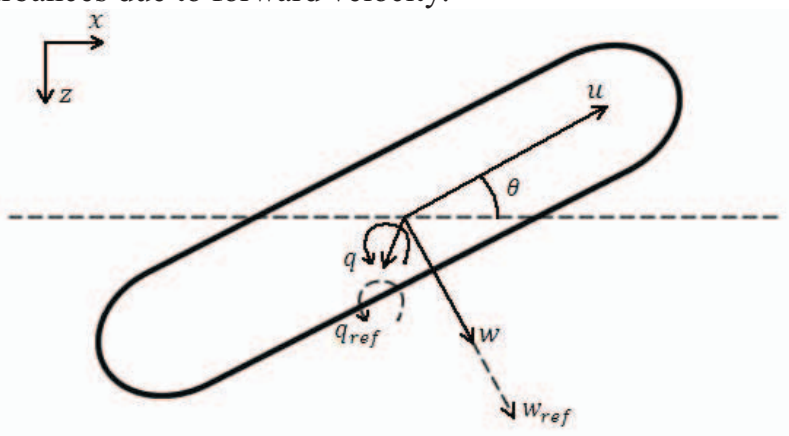

Fig. 3: Vertical motion of MARES

We wish to control the linear and angular velocities $w$ and $q$, whereby we define an error vector as follow:

$$
\begin{aligned}
e & =v_{r}-v_{\text {ref }} \\
& =\left[\begin{array}{c}
u-u_{\text {ref }} \\
w-w_{\text {ref }} \\
q-q_{\text {ref }}
\end{array}\right] \\
& =\left[\begin{array}{c}
0 \\
w-w_{\text {ref }} \\
q-q_{\text {ref }}
\end{array}\right] .
\end{aligned}
$$

where $e, v_{r}, v_{\text {ref }}=\left[\begin{array}{lll}u_{\text {ref }} & w_{\text {ref }} & q_{\text {ref }}\end{array}\right]^{T} \in \mathbb{R}^{3}$, in this case.

It is important to refer that the error component after $x_{B}$ $\left(u-u_{r e f}\right)$ is always zero because we are not interested in controlling this variable.

The expression (6) defines the Lyapunov candidate function:

$$
V=\frac{1}{2} e^{T} e
$$

whose time derivative results

$$
\begin{aligned}
\dot{V} & =e^{T} \dot{e} \\
& =e^{T}\left(\dot{v}_{r}-\dot{v}_{r e f}\right) .
\end{aligned}
$$

After the Lyapunov theory, the stability of the system implies that $\dot{V}$ must be negative definite, which leave to impose

$$
e^{T}\left(\dot{v}_{r}-\dot{v}_{r e f}\right)<0 \quad \Longrightarrow \quad \dot{v}_{r}-\dot{v}_{r e f}<-k_{e} e,
$$

where $k_{e} \in \mathbb{R}, k_{e}>0$.

In order to satisfy (8), knowing that we can not vary the error variable $e$ instantaneously, we define a new error variable $\alpha$, which we want to leave to zero, such that

$$
\begin{gathered}
\alpha=\dot{v}_{r}-\dot{v}_{r e f}+k_{e} e \\
\mathbb{\mathbb { }} \\
\dot{v}_{r}=\alpha+\dot{v}_{r e f}-k_{e} e .
\end{gathered}
$$

Re-writing the time derivative of the Lyapunov function, we get

$$
\dot{V}=e^{T}\left(\alpha-k_{e} e\right) .
$$

Substituting (9) into the simplified equation of (4) and manipulating algebraically, we obtain, assuming that $M=$ $M_{R B}+M_{A}$ is invertible,

$$
\begin{array}{r}
\alpha=M^{-1}\left[-\left(C_{R B}\left(v_{r}\right)+C_{A}\left(v_{r}\right)+D_{V}\left(v_{r}\right)\right) \cdot v_{r}\right. \\
\left.-g(\eta)-M\left(\dot{v}_{r e f}-k_{e} e\right)+P f_{p}\right],
\end{array}
$$

where, in this case, $C_{R B}, C_{A}, D_{V}, M \in \mathbb{R}^{3 \times 3}, g$, $\dot{v}_{\text {ref }} \in \mathbb{R}^{3}$, $f_{p} \in \mathbb{R}^{2}$ and $P \in \mathbb{R}^{3 \times 2}$ are matrices and vectors of the simplified model.

In order to guarantee that $\dot{V}$ is negative definite, we choose $f_{p}$ as we show in the following expression, considering that we can actuate directly in this variable:

$$
\begin{gathered}
f_{p}=P^{+}\left[\left(C_{R B}\left(v_{r}\right)+C_{A}\left(v_{r}\right)+D_{V}\left(v_{r}\right)\right) \cdot v_{r}+g(\eta)\right. \\
\left.+M\left(\dot{v}_{r e f}-k_{e} e\right)\right]
\end{gathered}
$$

where $P^{+}$is the generalized inverse of $P$. This expression gives the closed loop control law for the vertical velocity.

Consequently, the time derivative of the Lyapunov function in (10) results

$$
\dot{V}=-k_{e} e^{T} e<0, \quad \forall e \neq 0,
$$

as desired for the asymptotical stability of the system.

The determination of the value of $k_{e}$ depends on actuators characteristics, especially on their saturation value. This gain is dimensioned in order to not reach actuation saturation during long intervals of time, which could degrade the response of the system. In addition, it is convenient to define error saturations, preventing the non desirable saturation reaching. These saturations are determined according to maximum obtainable velocities.

In most of cases, controller gains must be adjusted using practical techniques, in particular for nonlinear systems, for which it is difficult to compute response characteristics as overshoot, rising time or settling time.

\section{B. Horizontal velocity controller}

As for the previous case, we start by reducing the order of the model in (4), reducing the complexity of the controller determination. Only motions after $x_{B}, y_{B}$ and yaw are considered, as it is shown in fig. 4. Remaining components are neglected because, according to the model [9], their influences are relatively small, whereby their inclusion is not justified and are considered disturbances. This implies that third, fourth and fifth line and columns of (4) are eliminated. In vectors cases, only lines are eliminated.

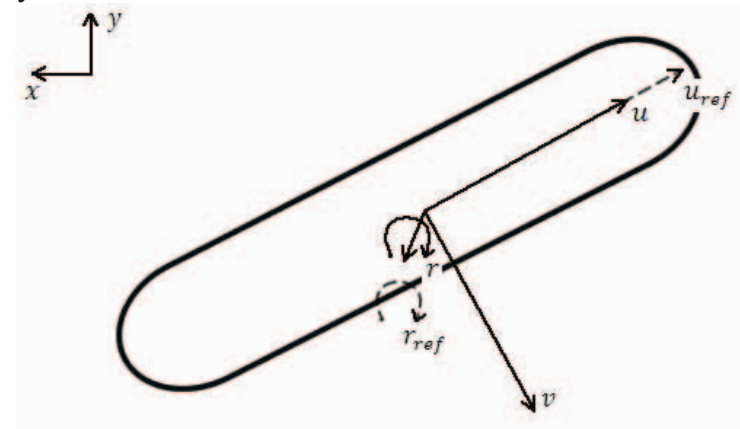

Fig. 4: Horizontal motion of MARES 
As it is illustrated in fig. 4, we are interested in controlling the forward velocity $u$ and the angular velocity $r$. Thus, we may define the error vector $e \in \mathbb{R}^{3}$ as the difference between the velocity reference $v_{\text {ref }}$ and the relative velocity $v_{r}$ :

$$
\begin{aligned}
e & =v_{r}-v_{\text {ref }} \\
& =\left[\begin{array}{l}
u-u_{\text {ref }} \\
v-v_{\text {ref }} \\
r-r_{\text {ref }}
\end{array}\right] \\
& =\left[\begin{array}{c}
u-u_{\text {ref }} \\
0 \\
r-r_{\text {ref }}
\end{array}\right] .
\end{aligned}
$$

Note that the lateral velocity error is always null, since we do not want to control it.

The determination of this controller is similar in every step to the vertical velocity controller. The resulting expression of the control law is equal to (12). Only matrices and vectors of the simplified model are different.

Computing of the gain is either similar to the previous section, where actuation saturations and maximum values of velocities are taken into account to define $k_{e}$ and error saturations values.

\section{Vertical position controller}

In this subsection we will present two different approaches to control vertical position $(z$ and $\theta)$. The first is based on Lyapunov theory and on backstepping techniques [11] while the second uses the vertical velocity controller deduced in $A$, with an external loop of position.

\section{Controller 1}

In order to simplify the manipulation of the controller expression, only motion after $x_{B}, z_{B}$ and pitch are considered. As for the vertical velocity controller, second, fourth and sixth lines are eliminated from the expression (4).

For the design of the controller, it is more interesting to define vertical position references in the earth-fixed referential. To relate vectors of the simplified model (coordinates in $x_{B}, Z_{B}$ and pitch components) in both referential, we must define a matrix $R(\theta) \in \mathbb{R}^{3 \times 3}$ such that

$$
\left[\begin{array}{c}
\dot{x} \\
\dot{z} \\
\dot{\theta}
\end{array}\right]=R(\theta)\left[\begin{array}{l}
u \\
w \\
q
\end{array}\right] .
$$

Considering that there is no motion after roll (rotation after $x_{B}$, $\phi=0)$, it results:

$$
\begin{aligned}
R(\theta) & =\left[\begin{array}{cc}
J(\theta)(1,3 ; 1,3) & 0_{2 \times 1} \\
0_{1 \times 2} & 1
\end{array}\right] \\
& =\left[\begin{array}{ccc}
\cos \theta & \sin \theta & 0 \\
-\sin \theta & \cos \theta & 0 \\
0 & 0 & 1
\end{array}\right]
\end{aligned}
$$

where $J(\theta)(1,3 ; 1,3)$ represents the result of the selection of first and third lines and columns of the rotation matrix in section II, assuming that $\phi$ and $\psi$ are zero.

In this case $\dot{\theta}=q$, which allows defining a new matrix that relates $R$ with its time derivative, such that

$$
\dot{R}(\theta)=S(q) \cdot R(\theta) \text {, }
$$

where $S(q) \in \mathbb{R}^{3 \times 3}$ is skew-symmetric, given by

$$
S(q)=\left[\begin{array}{ccc}
0 & q & 0 \\
-q & 0 & 0 \\
0 & 0 & 0
\end{array}\right]
$$

This relation will be useful in the next controller deducing steps.

We define the error vector in the body-fixed referential as:

$$
\begin{aligned}
e & =J^{T}\left(\eta-\eta_{\text {ref }}\right) \\
& =J^{T}\left[\begin{array}{l}
x-x_{\text {ref }} \\
z-z_{\text {ref }} \\
\theta-\theta_{\text {ref }}
\end{array}\right] \\
& =J^{T}\left[\begin{array}{c}
0 \\
z-z_{\text {ref }} \\
\theta-\theta_{\text {ref }}
\end{array}\right]
\end{aligned}
$$

Note that we do not want to control the $x$-component, so its error is always null $\left(x_{\text {ref }}=x\right)$. This leaves to following first and second time derivatives of the reference position, respectively:

$$
\begin{gathered}
\dot{\eta}_{r e f}=\left[\begin{array}{c}
0 \\
\dot{z}_{r e f} \\
\dot{\theta}_{r e f}
\end{array}\right]+\left[\begin{array}{ccc}
1 & 0 & 0 \\
0 & 0 & 0 \\
0 & 0 & 0
\end{array}\right] \cdot J \cdot v_{r} . \\
\ddot{\eta}_{r e f}=\left[\begin{array}{c}
0 \\
\dot{z}_{r e f} \\
\dot{\theta}_{r e f}
\end{array}\right]+\left[\begin{array}{ccc}
1 & 0 & 0 \\
0 & 0 & 0 \\
0 & 0 & 0
\end{array}\right] \cdot\left(S \cdot J \cdot v_{r}+J \cdot \dot{v}_{r}\right) .
\end{gathered}
$$

We now define the Lyapunov candidate function as follow:

$$
V_{1}=\frac{1}{2} e^{T} e
$$

Whose time derivative results

$$
\begin{aligned}
\dot{V}_{1} & =e^{T}\left[-S J^{T}\left(\eta-\eta_{\text {ref }}\right)+J^{T}\left(\dot{\eta}-\dot{\eta}_{\text {ref }}\right)\right] \\
& =e^{T}\left[-S e+J^{T}\left(\dot{\eta}-\dot{\eta}_{\text {ref }}\right)\right] .
\end{aligned}
$$

Knowing that $e^{T} S e=0$ because that $S$ is skew-symmetric, we get:

$$
\dot{V}_{1}=e^{T} J^{T}\left(\dot{\eta}-\dot{\eta}_{\text {ref }}\right)
$$

Backstepping techniques will be used in order to reach an adequate control law for the motion of the vehicle. Recursive error variables and Lyapunov candidate functions will be determined.

The asymptotical stability implies that $\dot{V}_{1}$ is negative definite, so we impose that:

$$
\begin{aligned}
\dot{V}_{1}<0 \quad \Rightarrow \quad J^{T}\left(\dot{\eta}-\dot{\eta}_{\text {ref }}\right) & <-k_{e} e \\
v_{r}-J^{T} \dot{\eta}_{\text {ref }} & <-k_{e} e,
\end{aligned}
$$

where $k_{e} \in \mathbb{R}^{+}$. Note that we consider $J^{T} \dot{\eta}=v_{r}$ because we assume that the inertial referential moves with the irrotational (no angular velocity) flow $v_{f}$, whose acceleration is considered null.

We define a new error variable that we want to leave to zero:

$$
\alpha_{1}=v_{r}-J^{T} \dot{\eta}_{r e f}+k_{e} e
$$

Substituting this last expression into (4), we obtain

$$
\begin{aligned}
M \dot{\alpha}_{1}=-\left(C_{R B}\left(v_{r}\right)\right. & \left.+C_{A}\left(v_{r}\right)+D_{V}\left(v_{r}\right)\right) \\
& \cdot\left(\alpha_{1}+J^{T} \dot{\eta}_{r e f}-k_{e} e\right)-g(\eta) \\
& +P f_{p} \\
& -M\left[-S J^{T} \dot{\eta}_{r e f}+J^{T} \ddot{\eta}_{r e f}\right. \\
& \left.-k_{e}\left(-S e+J^{T}\left(\dot{\eta}-\dot{\eta}_{r e f}\right)\right)\right] .
\end{aligned}
$$


From this expression, we may conclude that it will not be always possible to leave $\alpha_{1}$ to zero if the control law is deduced directly at this step. For example, if $v_{r}=0$ and $\dot{\eta}_{\text {ref }}=0$, it results $\dot{\alpha}_{1}=0$, which implies no variation in $\alpha_{1}$.

Thus, we define a new error variable that we want to leave to zero too:

$$
\alpha_{2}=\alpha_{1}-\delta
$$

where $\delta \in \square^{3}$ is constant vector with arbitrarily small components, in modulus. It is referred as a design vector by [7] and allows defining the steady state error signal.

The augmented Lyapunov function comes

$$
V_{2}=V_{1}+\frac{1}{2} \alpha_{2}^{T} M \alpha_{2}
$$

and its time derivative results

$$
\begin{aligned}
\dot{V}_{2} & =\dot{V}_{1}+\alpha_{2} M \dot{\alpha}_{2} \\
& =e^{T} \alpha_{2}+e^{T} \delta-k_{e} e^{T} e+\alpha_{2} M \dot{\alpha}_{2} .
\end{aligned}
$$

Substituting (28) into (27), we get

$$
\begin{aligned}
M \dot{\alpha}_{2}=-\left(C_{R B}\left(v_{r}\right)\right. & \left.+C_{A}\left(v_{r}\right)+D_{V}\left(v_{r}\right)\right) \\
& \cdot\left(\alpha_{2}+\delta+J^{T} \dot{\eta}_{r e f}-k_{e} e\right)-g(\eta) \\
& +P f_{p} \\
& -M\left[-S J^{T} \dot{\eta}_{r e f}+J^{T} \ddot{\eta}_{r e f}\right. \\
& \left.-k_{e}\left(-S e+J^{T}\left(\dot{\eta}-\dot{\eta}_{r e f}\right)\right)-\dot{\delta}\right] .
\end{aligned}
$$

Aiming to turn $\dot{V}_{2}$ negative definite, we choose the control variable such that

$$
\begin{aligned}
f_{P}=P^{+}\left[\left(C_{R B}\left(v_{r}\right)\right.\right. & \left.+C_{A}\left(v_{r}\right)+D_{V}\left(v_{r}\right)\right) \cdot v_{r}+g(\eta) \\
& +M\left[-S J^{T} \dot{\eta}_{r e f}+J^{T} \ddot{\eta}_{r e f}\right. \\
& \left.-k_{e}\left(-S e+J^{T}\left(\dot{\eta}-\dot{\eta}_{r e f}\right)\right)-\dot{\delta}\right] \\
& \left.-e-k_{\alpha_{2}}\left[v_{r}-J^{T} \dot{\eta}_{r e f}+k_{e} e-\delta\right]\right]
\end{aligned}
$$

where $k_{\alpha_{2}} \in \mathbb{R}^{+}$.

This allows to re-write (30) as

$$
\dot{V}_{2}=e^{T} \delta-k_{e} e^{T} e-k_{\alpha_{2}} \alpha_{2}^{T} \alpha_{2}
$$

Analyzing this last expression, we may verify that $\dot{V}_{2}$ could not be always negative definite due to the term $e^{T} \delta$, but $\delta$ can have arbitrary small values depending on the steady state error that we wish. In other hand, if the instability caused by this term leaves the system to diverge, $e$ will increase, in modulus, and consequently $\dot{V}_{2}$ will turn negative.

As for the previous controller gains, $k_{e}$ and $k_{\alpha_{2}}$ must be determined considering actuation limits and error saturation that either must be established.

\section{Controller 2}

Unlike to the vertical position controller 1, we will deduce a controller that uses the velocity controller deduced in $A$. The architecture of the system will result in that shown in fig.5.

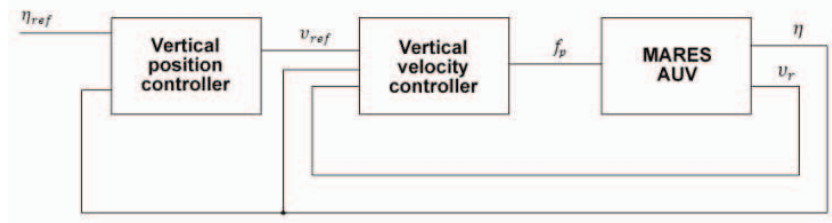

Fig. 5: Architecture of vertical position control
Velocity references $\left(w_{\text {ref }}\right.$ and $\left.q_{\text {ref }}\right)$ will be generated dynamically and will be applied to the vertical velocity controller. The use of this last controller allows virtual decoupling of both linear and angular velocities.

Consider the following figure. We wish that the vehicle reaches a depth reference $z_{\text {ref }}$ and a pitch angle $\theta_{\text {ref }}$.

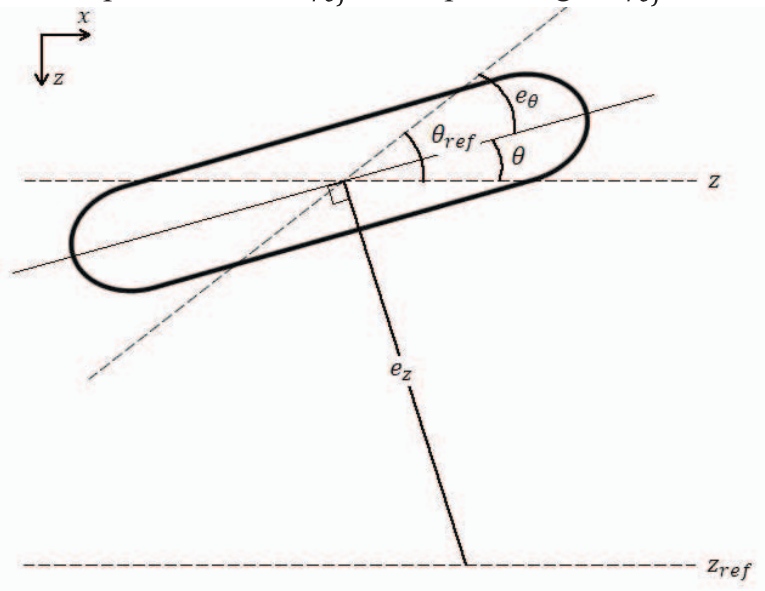

Fig. 6: Vertical motion in position control

To determine this controller, we start by defining position errors in the vertical plane (see fig.6):

$$
\begin{gathered}
e_{z}=\frac{1}{\cos \theta}\left(z-z_{\text {ref }}\right), \\
e_{\theta}=\theta-\theta_{\text {ref }} .
\end{gathered}
$$

These errors allow defining of a position controller with proportional and integral gains. In reality, this will be constituted by two independent controllers of the depth and of the angle:

$$
\begin{aligned}
& w_{\text {ref }}=-K_{p z} e_{z}-K_{i z} \int e_{z} d t, \\
& q_{\text {ref }}=-K_{p \theta} e_{\theta}-K_{i \theta} \int e_{\theta} d t .
\end{aligned}
$$

where $K_{p z}, K_{i z}, K_{p \theta}, K_{i \theta} \in \mathbb{R}^{+}$are proportional and integral gains.

With the determination of this controller, we want to obtain an invariant behavior independent of error values. However, integral terms in (36) and (37) are directly dependents of errors. To illustrate this concept, consider a constant error that leaves actuation to saturation during an interval of time. The maximum value of the velocity $w$ would be reached and the actuation would continue to be saturated. The bigger the error, the bigger the integral term due to the elapsed time and to error value. This behavior may be attenuated saturating the error, activating and reinitializing the integral component only when the error is not saturated:

$$
\int e d t=0, \quad|e|>e_{\max }
$$

Controller gains must be determined considering velocities saturation values of the vertical velocity controller and error saturations.

\section{Line following controller}

The controller that will be determined in this subsection will permit the following of an imaginary straight line in space 
defined by two horizontal points $\left(x_{1}, y_{1}\right)$ and $\left(x_{2}, y_{2}\right)$. With previous determined controllers we may assume that this motion is independent of the vertical one. Therefore, it implies that the vehicle can follow a line and dive simultaneously, for example. The architecture of the controller is presented in fig. 7.

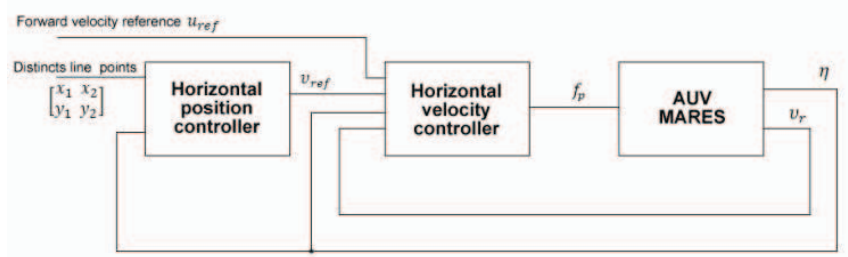

Fig. 7: Architecture of the line following controller

Note that forward relative velocity is defined independently. It will not be generated by the position controller and may be modified along the trajectory.

In order to guarantee a high dynamic response of the system for any distance and any angle to the line, it is necessary to determine an approach and a proximity controller. The first one will be responsible to approach the vehicle to the line while the second one will have the function of leave the error to zero in steady state. The commutation between them must be done according to the distance. The structure of the horizontal position controller in fig. 7 is presented in the following figure. Hysteresis values are arbitrated.

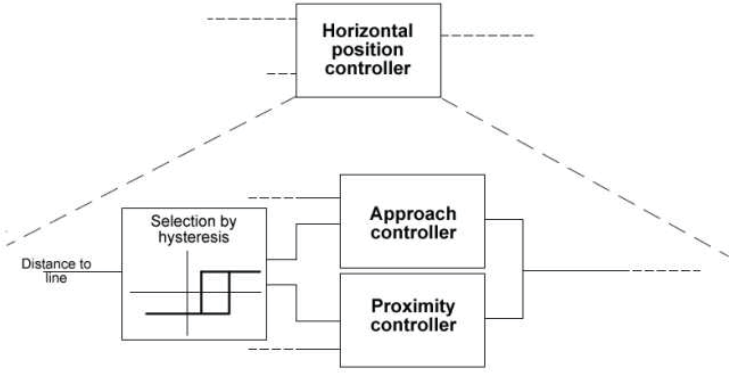

Fig. 8: Structure of the horizontal position controller

\section{Approach controller}

Imposing a forward velocity reference to the controller, it is necessary that its orientation (yaw angle $\psi$ ) allows the approach to the line. The minimum distance between the vehicle and the line is given by the segment that intersects the vehicle and is perpendicular to the line. Thus, the approach to the line will be done perpendicularly to the line.

Therefore, we define the equation of the straight line that we wish to follow as a function of the absolute position component $x$ :

$$
y_{r}(x)=m x+b,
$$

where $m=\frac{y_{2}-y_{1}}{x_{2}-x_{1}}$ and $b=y_{1}-m x_{1}$.

Assuming that $u>0$, we define the angle of the perpendicular to the line that we wish to follow as

$$
\psi_{p}=\left\{\begin{array}{cc}
\psi_{\text {ref }}-\frac{\pi}{2}, & y>y_{r} \\
\psi_{\text {ref }}+\frac{\pi}{2}, & y<y_{r}
\end{array}\right.
$$

where $\psi_{\text {ref }}=\operatorname{atan} 2\left(y_{2}-y_{1}, x_{2}-x_{1}\right)$.

Thus, the error of the angle of the vehicle during the approach to the line is given by

$$
e_{\psi}=\psi-\psi_{p}
$$

Presented concepts are shown in fig. 9.

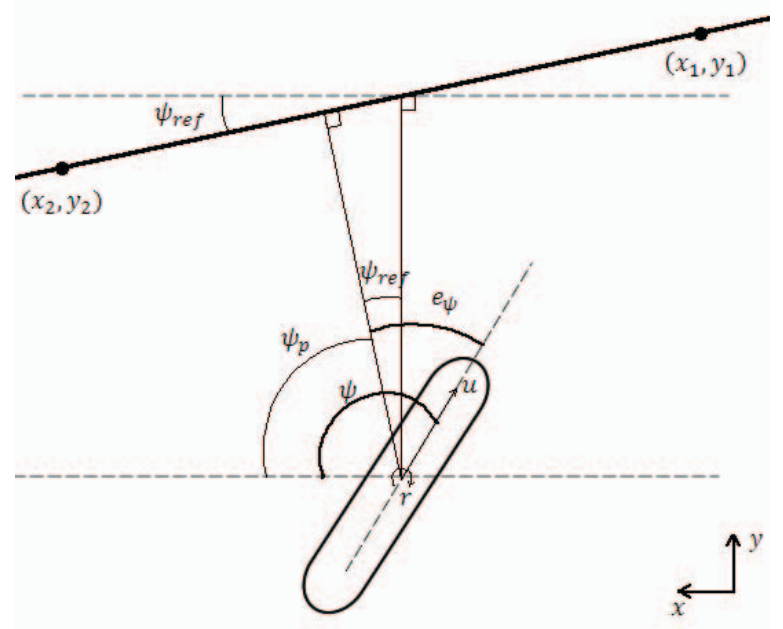

Fig. 9: Approach to the line

The resulting control law follows:

$$
r_{\text {ref }}=-K_{p \psi} e_{\psi} \text {. }
$$

where $K_{p \psi} \in \mathbb{R}^{+}$is the proportional gain computed taking into account the saturation of $e_{\psi}$ and the yaw velocity reference $r_{\text {ref }}$ saturation in the horizontal velocity controller, which it is applied.

\section{Proximity controller}

With this controller, we intend to obtain a null distance to the line in steady state. In order to reach this aim, we introduce a proportional and an integral component of the distance error, which is given by

$$
\begin{aligned}
e_{d} & =-\left(y-y_{r}\right) \cos \left(\pi-\psi_{\text {ref }}\right) \\
& =\left(y-y_{r}\right) \cos \left(\psi_{r e f}\right) .
\end{aligned}
$$

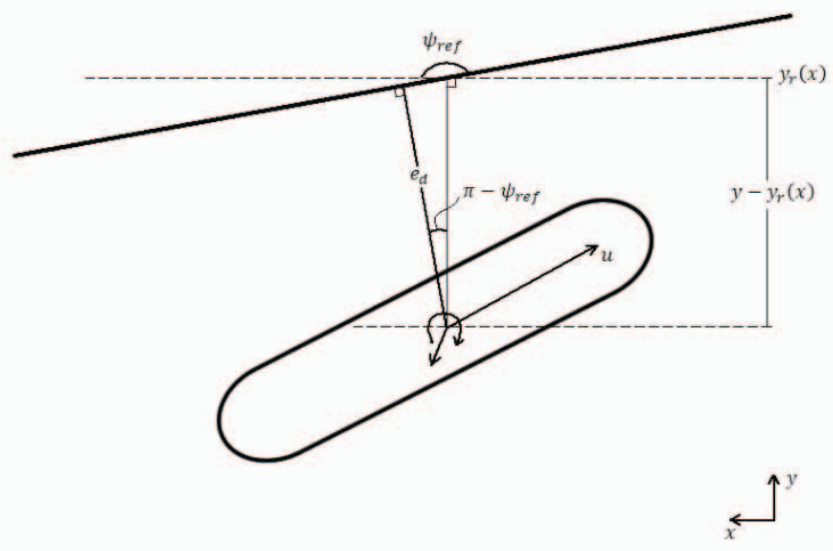

Fig. 10: MARES on the proximity of the line

In order to impose the following direction (from point 1 to point 2), it is also necessary adding a proportional term of the angle between the vehicle and the line. To satisfy this, we redefine the angle error:

$$
e_{\psi}=\psi-\psi_{\text {ref }}
$$


The control law results:

$$
r_{\text {ref }}=-K_{p \psi} e_{\psi}-K_{p d} e_{d}-K_{i d} \int e_{d} d t
$$

When there is a commutation to the approach controller, the integration must be suspended and reinitialized $\left(\int e_{d} d t=0\right)$.

Gains have to be computed in such a way that the $K_{p \psi} e_{\psi}$ is dominant and $K_{i d} \int e_{d} d t$ do not overcome $K_{p d} e_{d}$, in order to have an asymptotical convergence of the error to zero. The integral term is directly dependent of time and indirectly dependent of the forward velocity, whereby it results $K_{i d}(u)$. In other words, the integral gain must be computed for different $u$. Error saturations of the horizontal velocity controller must also be taken into account.

It is interesting that the vehicle could describe the same trajectory (relating to the fluid) during the line following, independently of the forward velocity $u$. The trajectory described by the vehicle in the position $(x, y)$ during an infinitesimal interval of time may be considered as an arc of circumference with curvature $r_{c}(x, y)$. If this function is invariant, that is

$$
\left.r_{c}(x, y)\right|_{u=u_{1}}=\left.r_{c}(x, y)\right|_{u=u_{2}},
$$

with $u_{1} \neq u_{2}$, then the trajectory will be invariant, since the initial condition are the same.

We may write

$$
r_{c}(x, y)=\frac{u_{1}(t)}{r_{1}(t)}=\frac{u_{2}(t)}{r_{2}(t)}
$$

where $r_{1}$ and $r_{2}$ are velocities of rotation after yaw for the velocities $u_{1}$ and $u_{2}$ respectively.

Control laws for both cases are given by

$$
\begin{aligned}
& \left.r_{r e f}\right|_{u=u_{1}}=-K_{p \psi} e_{\psi}-K_{p d} e_{d}-K_{i d} \int e_{d} d t, \\
& \left.r_{r e f}\right|_{u=u_{2}}=-K_{p \psi}^{\prime} e_{\psi}-K_{p d}^{\prime} e_{d}-K_{i d}^{\prime} \int e_{d} d t .
\end{aligned}
$$

Using (47), (48) and (49), we easily conclude that gains values are related as follow:

$$
\begin{gathered}
K_{p \psi}^{\prime}=\frac{u_{2}}{u_{1}} K_{p \psi} ; \quad K_{p d}^{\prime}=\frac{u_{2}}{u_{1}} K_{p d} ; \\
K_{i d}^{\prime}(u)=\frac{u_{2}}{u_{1}} K_{i d}(u) .
\end{gathered}
$$

\section{E. Circle following controller}

In this subsection, we intend to design a controller that allows following a circumference in the horizontal plane, defined by its center $\left(x_{C}, y_{C}\right)$ and its radius $r_{C}$. Many concepts presented here are similar to those presented for the design of the previous controller.

The architecture followed is equal to the presented in fig. 7, except in a detail: references applied to the horizontal position controller are the radius and the center of the circumference instead of line points. Here, we also need two "subcontrollers": an approach and a proximity controller.

\section{Approach controller}

We start by defining the error due to the distance to the circle:

$$
e_{d}=\sqrt{\left(x_{C}-x\right)^{2}+\left(y_{C}-y\right)^{2}}-r_{C}
$$

The approach to the circle is carried out controlling the yaw angle $\psi$, whose reference value is given by (52). If the vehicle is in the interior of the circle, it must be orientated to its exterior and vice-versa.

$$
\psi_{\text {ref }}= \begin{cases}\operatorname{atan} 2\left(x_{C}-x, y_{C}-y\right), & e_{d}>0 \\ \operatorname{atan} 2\left(x_{C}-x, y_{C}-y\right)+\pi, & e_{d}<0 .\end{cases}
$$

The angle error comes

$$
e_{\psi}=\psi-\psi_{\text {ref }}
$$

and the respective control law is given by:

$$
r_{r e f}=-K_{p \psi} e_{\psi} \text {. }
$$

When the vehicle reaches a sufficiently small (arbitrated) distance to the circle, there is a commutation to the proximity controller.

\section{Proximity controller}

The following figure illustrates the circle following maneuver.

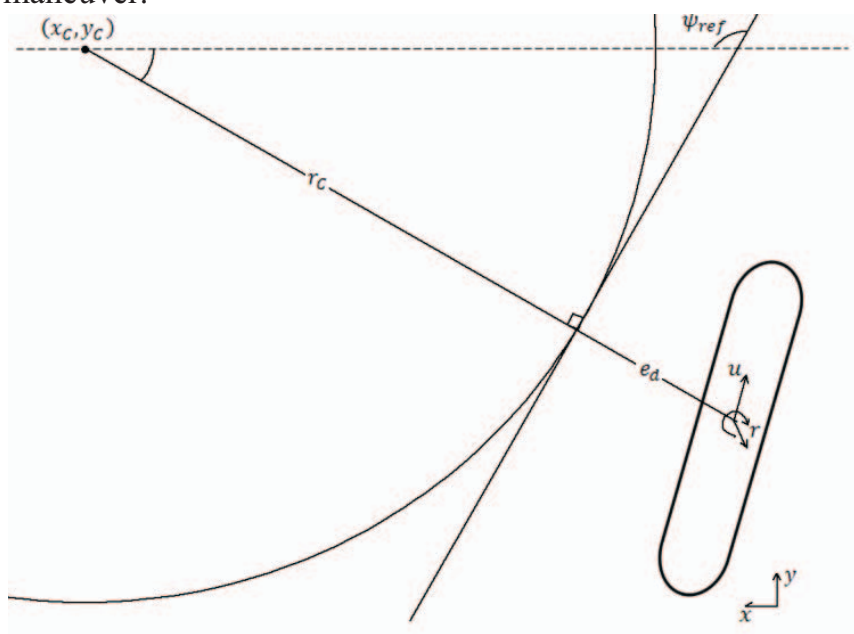

Fig. 11: MARES on the proximity of the circumference

The yaw angle reference is given by the following expression:

$$
\psi_{\text {ref }}= \begin{cases}\begin{array}{l}
\operatorname{atan} 2\left(x_{C}-x, y_{C}-y\right)+\pi / 2 \\
\text { counter clockwise rotation }
\end{array} & , \text { for } \\
\begin{array}{l}
\text { atan } 2\left(x_{C}-x, y_{C}-y\right)-\pi / 2 \\
\text { clockwise rotation. }
\end{array} & , \text { for }\end{cases}
$$

The yaw error expression results the same as in (53).

In order to obtain a null error of the distance in steady state, it is necessary to introduce proportional and integral components of it. Distance error is defined as we show in the next expression:

$$
e_{d}= \begin{cases}\sqrt{\left(x_{C}-x\right)^{2}+\left(y_{C}-y\right)^{2}}-r_{C} & , \text { for } \\ \text { counter clockwise rotation } & \\ -\left(\sqrt{\left(x_{C}-x\right)^{2}+\left(y_{C}-y\right)^{2}}-r_{C}\right), & \text { for } \\ \text { clockwise rotation. }\end{cases}
$$

We obtain the following control law:

$$
r_{r e f}=-K_{p \psi} e_{\psi}-K_{p d} e_{d}-K_{i d} \int e_{d} d t
$$


Gains are determined as it is referred for the line following case. It is also possible to achieve same trajectories applying expressions deduced in (50). Integral component must be reinitialized and maintained equal to zero when the approach controller is activated.

\section{F. Horizontal position controller: go to $\left(x_{\text {ref }}, y_{\text {ref }}\right)$}

Considering that the flow velocity of the involving fluid $v_{f}$ is sufficiently small compared to that of the MARES, in such a way that it can move in all directions, we determine a controller that supplies the horizontal velocity controller with a reference. The architecture adopted is similar to the one presented for the vertical position controller in fig. 7. It will be divided into two basic controllers: an approach controller similar to those presented in previous subsections and a proximity controller that allows the vehicle to stabilize in the target, with no motion regarding to an earth-fixed referential.

\section{Approach controller}

In order to guarantee that the system has a good behavior for a large range of operation, an approach is performed before the maneuver of immobilization. This is achieved at an externally defined forward velocity reference $u_{r e f}$, correcting the angle between the orientation of the vehicle and the straight formed by the reference point $\left(\mathrm{x}_{\mathrm{ref}}, \mathrm{y}_{\mathrm{ref}}\right)$ and the vehicle, along the trajectory. Thus, it result

$$
\psi_{\text {ref }}=\operatorname{atan} 2\left(y_{\text {ref }}-y, x_{\text {ref }}-x\right) \text {. }
$$

The control law is then given by

$$
r_{r e f}=-K_{p \psi} e_{\psi}
$$

where $e_{\psi}=\psi-\psi_{\text {ref }}$ and $K_{p \psi} \in \mathbb{R}^{+}$.

\section{Proximity controller}

Taking into account that MARES has only four degrees of freedom, in steady-state the vehicle must be parallel to the current flow. In fig. 12, we show the vehicle with a given reference $\left(x_{r e f}, y_{r e f}\right)$, in a fluid with non null linear velocity.

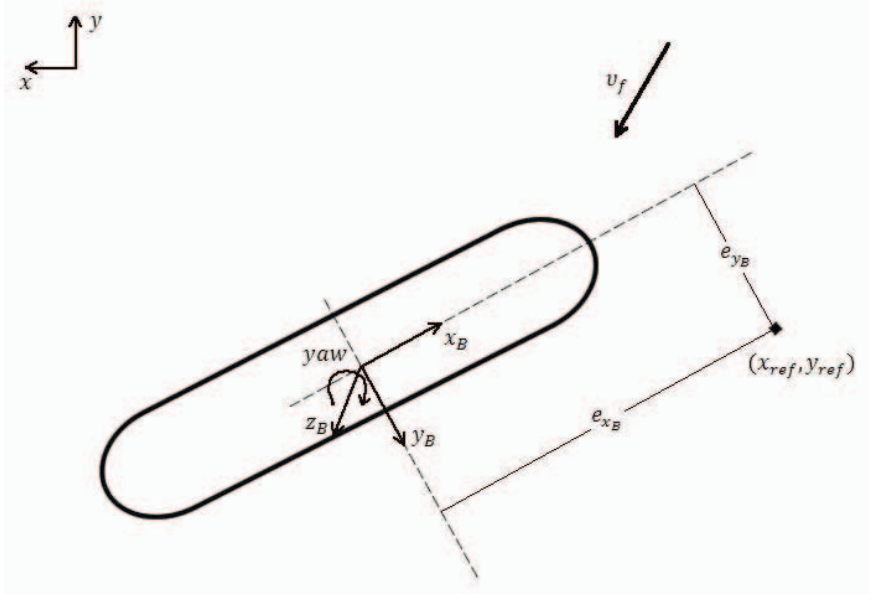

Fig. 12: Horizontal motion of MARES with non null current

The $y_{B}$-component of fluid velocity in the vehicle referential is given by $e_{v_{f}}$ :

$$
e_{v_{f}}=\left[\begin{array}{lll}
0 & 1 & 0
\end{array}\right] \cdot J^{T}(\psi) \cdot v_{f}
$$

which expression, for $r \approx 0$ and $p \approx 0$, may be approximated by

$$
e_{v_{f}} \approx\left[\begin{array}{lll}
0 & 1 & 0
\end{array}\right] \cdot J^{T}(\psi) \cdot \dot{\eta},
$$

where we recall that $\dot{\eta}$ is the time derivative of the absolute position in the inertial referential (earth fixed).

The error distance vector $\left(e_{x_{B}}, e_{y_{B}}\right)$ referred to the bodyfixed referential is given by the following expression:

$$
\left[\begin{array}{c}
e_{x_{B}} \\
e_{y_{B}} \\
0
\end{array}\right]=J^{T} \cdot\left(\left[\begin{array}{l}
x \\
y \\
0
\end{array}\right]-\left[\begin{array}{c}
x_{r e f} \\
y_{r e f} \\
0
\end{array}\right]\right)
$$

The effect of the longitudinal error $e_{x_{B}}$ must be reflected directly in the forward velocity reference $u_{r e f}$. In other hand, the lateral component of the fluid velocity $e_{v_{f}}$ and lateral component of the distance error $e_{y_{B}}$ must be actuate in the yaw velocity reference $r_{\text {ref }}$. Therefore, obtain the following control law:

$$
\begin{gathered}
u_{r e f}=-K_{p x} e_{x_{B}}-K_{i x} \int e_{x_{B}} d t \\
r_{r e f}=-K_{p v} e_{v_{f}}-K_{p y} e_{y_{B}}-K_{i y} \int e_{y_{B}} d t .
\end{gathered}
$$

where $K_{p x}, K_{i x}, K_{p v}, K_{p y}, K_{i y} \in \mathbb{R}^{+}$.

It is important to refer that, for the expression (64), the compensation effect of the flow, given by $K_{p v} e_{v_{f}}$, must be dominant relatively to others, guaranteeing that the fluid velocity do not induce an excessive lateral force. In other words, the yaw angle $\psi$ of the vehicle must oppose to the flow $\left(\psi \approx \operatorname{atan} 2\left(v_{f}, u_{f}\right)+\pi\right)$, with small deviations. So proportional and integral gains must be computed considering these facts and velocity error saturations of the horizontal velocity controller.

Integration components of (63) and (64) must be reinitialized whenever the approach controller is activated.

\section{SIMULATION AND EXPERIMENTAL RESULTS}

In order to test controllers determined, we implement a simulation of vehicle. The complete model with six degrees of freedom, deduced in [9], given by the nonlinear differential equation (4), is simulated through the Matlab Simulink. Environment restrictions and limitations are recreated: high uncertainty and low frequency due to the horizontal acoustic positioning [13] are the more important characteristics. In the case of MARES, positioning is done at a frequency of $0.5 \mathrm{~Hz}$ and is affected by an uncertainty of 1 to 2 meters. Remaining state variables, as depth, yaw and pitch angles are read directly from relative sensors and are assumed to be precise and not affected by noise.

In some cases, we intend to validate controllers and the dynamical model through comparing of results.

\section{A. Vertical motion}

Aiming to do different positions, we design a mission for the vehicle where it dives at constant velocity $w=0.5 \mathrm{~m} / \mathrm{s}$ at $\theta=0$ until reach $1.5 \mathrm{~m}$ of depth. At this moment, the position must be controlled at the same depth assuming several pitch angles. The response of the system is shown in fig. 13-14.

It can be seen that wave forms have different phases, particularly from $t=100 \mathrm{~s}$ to next instants of time. This is due 
to lack of synchronism of time between simulator and platform where controllers run.

In this case, we opt to show only vertical position controller 2 because this is what presents better performance. Controller 1 is particularly sensible to actuation saturation and, for fast dynamics, gives more poor performances than controller 1 . In addition, given that it has not integral component of the error, it do not reach null error in steady state.

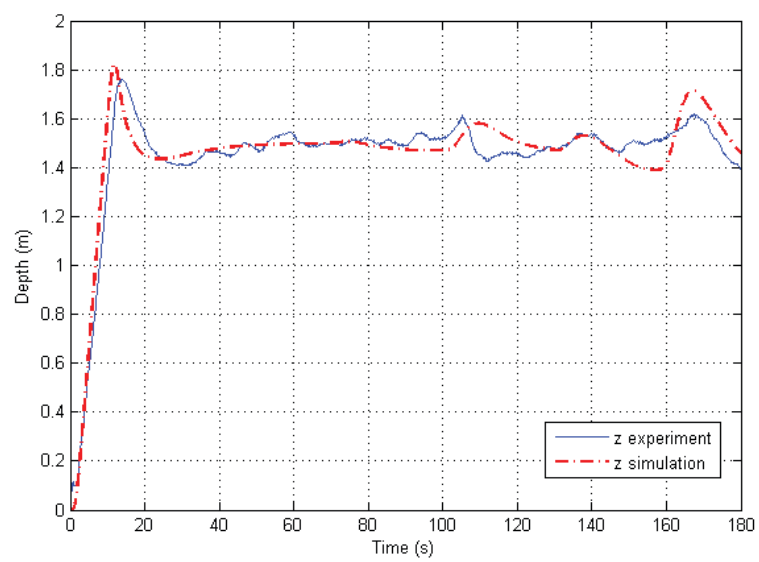

Fig. 13: Experimental and simulation results for depth $z$

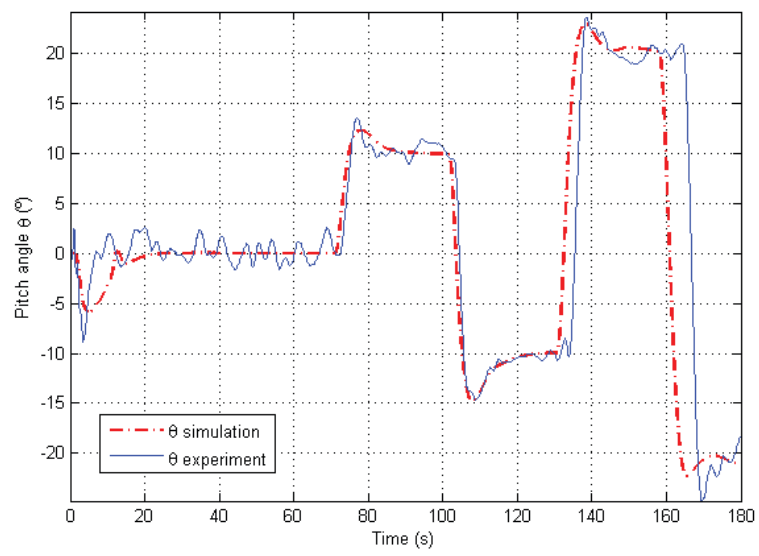

Fig. 14: Experimental and simulation results for pitch angle $\theta$

These results are satisfactory and allow validating of vertical velocity and position controllers. Model may be partially validated here, considering that experimental and simulated responses are very similar.

The additional noise in experimental collected data may be due to waves and wind that are not considered in the model and knowing that the vehicle is near from the surface.

\section{B. Line following}

In fig.15, the resulting trajectory for a mission where the vehicle must follow a line at $u=0.5 \mathrm{~m} / \mathrm{s}$ is shown.

As we can see, the trajectory affected by noise, particularly in the approach to the line with equation $y=50 \mathrm{~m}$, due to the acoustic localization. At the moment we are not in condition to verify if all collected points are correct because acoustic may fail during the operation. In these instants, estimators compute the position of the vehicle. However, it is possible to conclude that the general behavior is satisfactory.

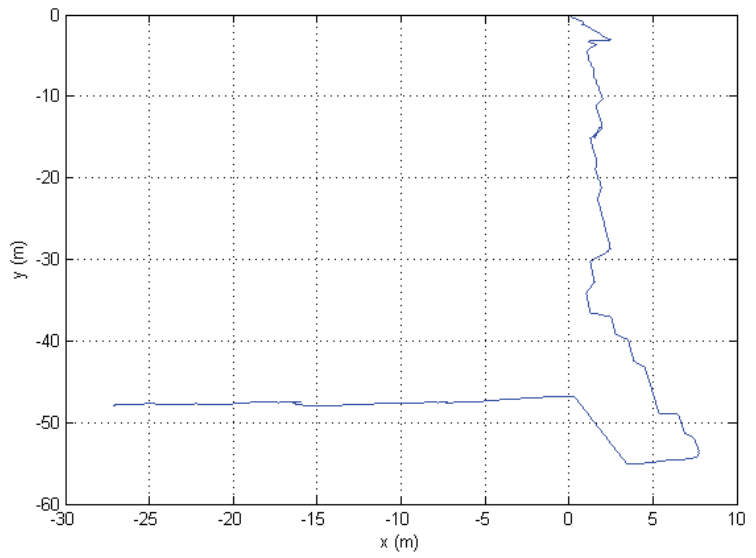

Fig. 15: Described trajectory for the line following

We show the filtered data of the velocity during the approach to the line in the next figure. This is obtained by the time derivative of the absolute position of the MARES AUV.

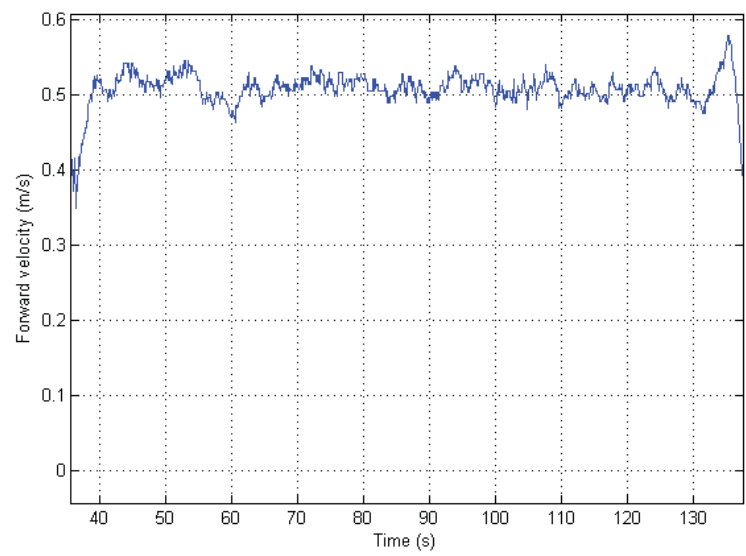

Fig. 16: Forward velocity during the line following

Through simulation, we obtain errors of the distance to the line as function of time for distinct velocities. This allows comparing trajectories in the approach to the line.

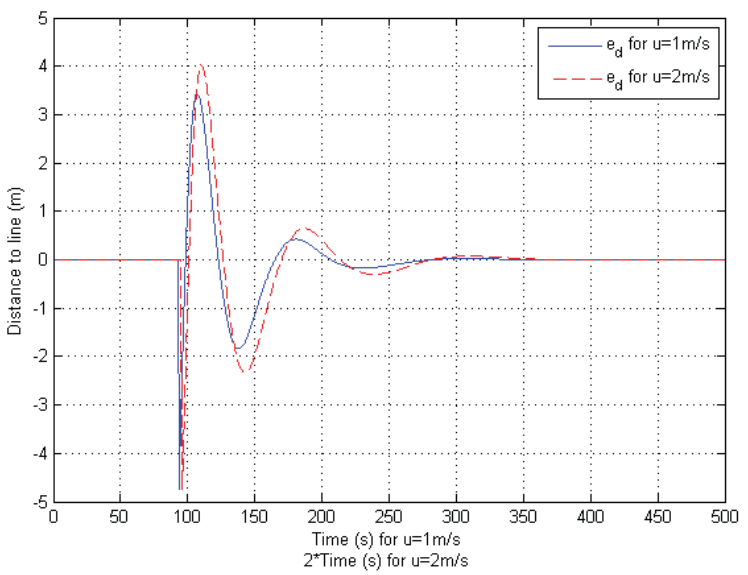

Fig. 17: Comparison between error for different forward velocities 
Though not exactly the same, errors are very close. It allows us to conclude that trajectories are very close too. Note that for initial instants the error is null because it is not considered during the approach to the line.

\section{Circle following}

In Fig. 18, we show the described trajectory for a circle following and with radius $r_{C}=5 \mathrm{~m}$ and center $\left(x_{C}, y_{C}\right)=$ $(25,0) \mathrm{m}$, at constant depth $z=25 \mathrm{~m}$. The simulated flow velocity is $v_{f}=[0.2,0.3,0] \mathrm{m} / \mathrm{s}$.

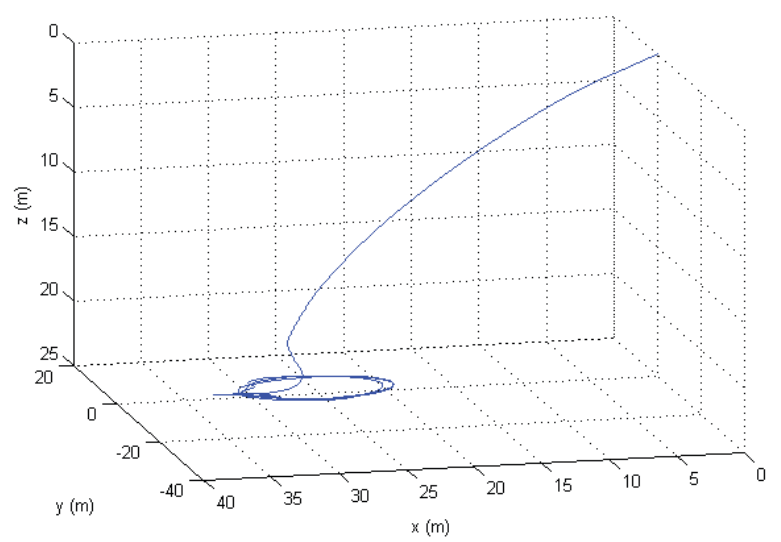

Fig. 18: Trajectory described by MARES for the circle following

\section{Immobilization}

For the demonstration of the immobilization controller, we simulate its behavior and consider a vertical position controller variant where only the pitch angle $\theta$ is controlled. This implies that the vehicle dives if it has negative $\theta$ and positive relative velocity $u$, as it shown in the next figure of the described trajectory.

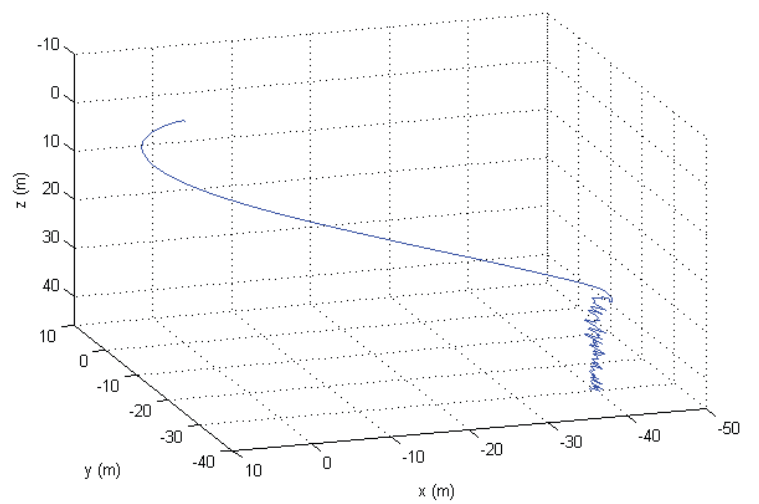

Fig. 19: Trajectory described for the horizontal immobilization with simulated noise.
In steady state, it is possible to verify that the vehicle position vary due to the positioning noise.

\section{CONCLUSIONS}

In this paper, we have started with background theory as kinematic, modeling and Lyapunov fundamental concepts, methods and theorems. Next, we have determined several controllers. Due to actuators configuration, we achieve decoupled motions of the MARES AUV, through control of horizontal and vertical positions and velocities. The implementation of these controllers allows performing relatively precise maneuvers such as follow an imaginary line or circle and immobilizing in a horizontal point. Finally, their performances are demonstrated by near from reality simulations and experiments, in some cases.

\section{REFERENCES}

[1] A. Matos and N. Cruz, Underwater Vehicles, Chapter 17. Austria: InTech, 2009

[2] N. Cruz and A. Matos, "The MARES AUV, a Modular Autonomous Robot for Environment Sampling". Québec, Canada : Proceedings of the MTS-IEEE Conference Oceans'2008, 2008.

[3] T. I. Fossen, Guidance and control of ocean vehicles. Chichester, England : John Wiley and Sons, 1994.

[4] O. M. Faltinsen, Hydrodynamic of high speed marine vehicle Cambridge, USA: Cambridge University Press, 2005.

[5] F. M. White, Fluid mechanics. Boston, USA: McGraw-Hill, 1999.

[6] Prestero, T. Verification of a Six-Degree of Freedom Simulation Model for the REMUS Autonomous Underwater Vehicle, MSc Thesis. MIT, Massachussets, 2001.

[7] A. P. Aguiar and J. P. Hespanha, "Trajectory-Tracking and PathFollowing of Underactuated Autonomous Vehicles with Parametric Modeling Uncertainty". IEEE transaction on automatic control, Vol. 52, no. 8, pp. 1362-1379, 2007

[8] R. Siegwart and I. R. Nourbakhsh, Introduction to Autonomous Mobile Robots. Cambridge, USA: MIT Press, 2004.

[9] B. Ferreira, M. Pinto, A. Matos and N. Cruz, "Modeling and motion analysis of the MARES autonomous underwater vehicle". Biloxi, USA: Proceedings of the MTS-IEEE Conference Oceans'09, 2009.

[10] J. J. Slotine and W. Li, Applied nonlinear control. Upper Saddle River: Prentice Hall, 1991.

[11] H. K. Khalil, Nonlinear Systems. Upper Saddle River, NJ: PrenticeHall, 2002.

[12] K. Ogata, Modern control engineering. New Jersey : Prentice Hall International, 1997.

[13] J. Borenstein, H. R. Everett and L. Feng, Where am I - systems and methods for mobile robot positioning. Michigan: Edited by J. Borenstein, University of Michigan, 1996. 